\title{
Some notes on conformable fractional Sturm-Liouville problems
}

Wei-Chuan Wang ${ }^{1 *}$

\section{"Correspondence:}

wangwc72@gmail.com;

wangwc@nqu.edu.tw

${ }^{1}$ Center for General Education,

National Quemoy University, 892

Kinmen, Taiwan, ROC

\section{Springer}

\begin{abstract}
The conformable fractional eigenvalue problem$$
-D_{x}^{\alpha} D_{x}^{\alpha} y+q(x) y=\lambda \rho(x) y
$$

is considered. We employ an easy and efficient method to derive its eigenvalue asymptotic expansion. On the basis of this result, we also investigate Ambarzumyan problems related to this eigenvalue problem as an application.
\end{abstract}

Keywords: Conformable fractional Sturm-Liouville problem; Eigenvalue expansion; Prüfer-type substitution; Ambarzumyan problem

\section{Introduction}

Fractional calculus is a generalization of integer differential calculus, allowing to define integrals and derivatives of real or complex order. Various types of fractional derivatives, such as Riemann-Liouville, Caputo, Grunwald-Letnikov, Riesz and Weyl, have been introduced. All of them are defined via fractional integrals, thus they inherit nonlocal properties from integral. This leads to failure to satisfy some of the basic properties owned by usual derivatives, e.g., the product rule, chain rule, Rolle's theorem, mean value theorem, and composition rule. These inconsistencies come with difficulties and inconvenience in mathematical analysis. For this reason, some scholars introduced the concept of local fractional derivatives [5, 9, 12, 14, 20]. In 2014, Khalil et al. introduced a new local fractional derivative, called the conformable fractional derivative (CFD) [13]. Now this subject is under strong development, see $[2,7,10,11]$ and the references therein. Later, Al-Refai and Abdeljawad [6] investigated the conformable fractional Sturm-Liouville problem (CFSLP) and showed that the eigenvalues are real and simple and the eigenfunctions corresponding to distinct eigenvalues are orthogonal. Also, they established a fractional Rayleigh quotient result that can be used to estimate the first eigenvalue.

However, some authors have argued that conformable fractional derivative is not a truly fractional operator [1, 16-19]. And the physical interpretation and some potential applications of this derivative seem not solid. In [8], the authors pointed out that the conformable derivative really results from the ordinary derivative with the change of $x \rightarrow \frac{x^{\alpha}}{\alpha}$

(c) The Author(s) 2021. This article is licensed under a Creative Commons Attribution 4.0 International License, which permits use sharing, adaptation, distribution and reproduction in any medium or format, as long as you give appropriate credit to the original author(s) and the source, provide a link to the Creative Commons licence, and indicate if changes were made. The images or other third party material in this article are included in the article's Creative Commons licence, unless indicated otherwise in a credit line to the material. If material is not included in the article's Creative Commons licence and your intended use is not permitted by statutory regulation or exceeds the permitted use, you will need to obtain permission directly from the copyright holder. To view a copy of this licence, visit http://creativecommons.org/licenses/by/4.0/. 
when applied to differential functions. They also tried to contribute to the physical interpretation when these derivatives are applied to physics and engineering. In another recent paper [21], the authors provided a good account of heredity/nonheredity and locality/nonlocality. They introduced a general conformable fractional derivative (GCFD) and gave the physical and geometrical interpretations of GCFD. The above is done by a new framework named extended Gâteaux derivative and linear extended Gâteaux derivative which are natural extensions of Gâteaux derivative. So, in our opinion, the exploration of the conformable derivative and its generalizations can still be interesting and valuable.

In this note we consider the CFSLP

$$
\begin{aligned}
& -D_{x}^{\alpha} D_{x}^{\alpha} y+q(x) y=\lambda \rho(x) y, \quad \text { on }(0,1), \\
& y(0)=y(1)=0,
\end{aligned}
$$

where $\lambda$ is the spectral parameter and $D_{x}^{\alpha}$ is the conformable fractional derivative of order $\alpha$. In 2020, Mortazaasl and Akbarfam [15] developed some spectral theories for the CFSLP with $\rho \equiv 1$ and deduced the asymptotic behavior of eigenvalues and eigenfunctions by analyzing the conformable fractional Volterra integral equations. They also showed that the eigenvalues of the CFSLP with separated boundary conditions are all real and simple, and the $n$th eigenfunction has precisely $n-1$ nodes in the interval $(0,1)$ for sufficiently large $\mathrm{n}$. Also in [4], uniqueness theorems for the solutions of inverse problems according to the Weyl function, two given spectra, classical spectral data, and mixed data are studied. Ambarzumyan-type problems are investigated for the conformable fractional Sturm-Liouville operator in [3]. Motivated by the idea in [3, 4, 6, 15], we intend to give an alternative proof (an easy method) for eigenvalue asymptotic estimates and acquire an application of Ambarzumyan problems as a consequence. For $q \equiv 0$ and $\rho \equiv 1$, it is known that the eigenfunctions of (1) are $\sin \left(n \pi x^{\alpha}\right)$ and the corresponding eigenvalues are $(\alpha n \pi)^{2}$ via a direct calculation. Note that, for $q \equiv 0$ and $\alpha=1,(1)$ is a string equation and $\rho$ is called a density function. For general $q$ and $\rho$, we plan to apply an easy and efficient method, the Prüfer angle approach, to achieve our goal. Throughout this note we assume that the following conditions hold:

$\left(C_{1}\right) \alpha \in\left(\frac{1}{2}, 1\right], q \in C[0,1]$

$\left(C_{2}\right) \rho>0$ with $\rho \in C^{2}(0,1)$.

The following is the first result.

Theorem 1.1 (Eigenvalue asymptotics)

(i) For the potential type of (1)-(2), $\rho \equiv 1$, the Dirichlet eigenvalue $\lambda_{n}$ satisfies

$$
\sqrt{\lambda_{n}}=\alpha n \pi+\frac{1}{2 n \pi} \int_{0}^{1} x^{\alpha-1} q(x) d x+o\left(\frac{1}{n}\right)
$$

for sufficiently large $n$.

(ii) For the density type of (1)-(2), $q \equiv 0$, the eigenvalue $\lambda_{n}$ satisfies

$$
\sqrt{\lambda_{n}} \int_{0}^{1} x^{\alpha-1} \rho^{1 / 2}(x) d x=n \pi+O\left(\frac{1}{n}\right)
$$

for sufficiently large $n$. 
In virtue of Theorem 1.1 and the fractional Rayleigh quotient result [6], we turn to study the Ambarzumyan problems related to (1). The following is the second result.

Theorem 1.2 (Ambarzumyan problems) Consider the potential type of (1), $\rho \equiv 1$.

(i) If the eigenvalues of (1)-(2) are $\lambda_{n}=(\alpha n \pi)^{2}, n \in \mathbb{N}$, and the function $q$ satisfies

$$
\int_{0}^{1} x^{\alpha-1} q(x) \cos \left(2 \pi x^{\alpha}\right) d x=0
$$

then $q \equiv 0$ on $(0,1)$.

(ii) If the Neumann eigenvalues of (1) coupled with $D_{x}^{\alpha} y(0)=D_{x}^{\alpha} y(1)=0$ are $\mu_{n}=[\alpha(n-1) \pi]^{2}$, then $q \equiv 0$ on $(0,1)$.

\section{Some preliminaries and proofs of the main results}

In this section, we first recall the elementary definitions and properties of conformable fractional calculus for the reader's convenience.

Definition 2.1 (cf. $[2,13]$ ) Let $0<\alpha \leq 1$ and $f:[0, \infty) \rightarrow \mathbb{R}$.

(i) The conformable fractional derivative of $f$ of order $\alpha$ at $x>0$ is defined by

$$
D_{x}^{\alpha} f(x)=\lim _{\epsilon \rightarrow 0} \frac{f\left(x+\epsilon x^{1-\alpha}\right)-f(x)}{\epsilon},
$$

and the conformable fractional derivative at 0 is defined as $D_{x}^{\alpha} f(0)=\lim _{x \rightarrow 0^{+}} D_{x}^{\alpha} f(x)$. Note that if $f$ is differentiable, then

$$
D_{x}^{\alpha} f(x)=x^{1-\alpha} f^{\prime}(x)
$$

where $^{\prime}=\frac{d}{d x}$ is the ordinary derivative with respect to $\mathrm{x}$. If $D_{x}^{\alpha} f\left(x_{0}\right)$ exists, one can say that $f$ is $\alpha$-differentiable at $x_{0}$.

(ii) The conformable fractional integral of $f$ of order $\alpha$ is defined by

$$
I_{\alpha} f(x)=\int_{0}^{x} t^{\alpha-1} f(t) d t \quad \text { for } x>0
$$

Proposition 2.2 (cf. [2, 13, 15])

(i) Let $f:[0, \infty) \rightarrow \mathbb{R}$ be any continuous function. Then, for all $x>0$, we have

$$
D_{x}^{\alpha} I_{\alpha} f(x)=f(x)
$$

(ii) Let $f:(0, b) \rightarrow \mathbb{R}$ be differentiable. Then, for $x>0$, we have

$$
I_{\alpha} D_{x}^{\alpha} f(x)=f(x)-f(0)
$$

(iii) For all $p \in \mathbb{R}, D_{x}^{\alpha}\left(x^{p}\right)=p x^{p-\alpha}$.

(iv) Let $f, g:(0, \infty) \rightarrow \mathbb{R}$ be $\alpha$-differentiable. Then

$$
D_{x}^{\alpha}(f g)=\left(D_{x}^{\alpha} f\right) g+f\left(D_{x}^{\alpha} g\right)
$$


and

$$
D_{x}^{\alpha}\left(\frac{f}{g}\right)=\frac{\left(D_{x}^{\alpha} f\right) g-f\left(D_{x}^{\alpha} g\right)}{g^{2}} \text { with } g \neq 0
$$

(v) ( $\alpha$-chain rule) Let $f, g:(0, \infty) \rightarrow \mathbb{R}$ be $\alpha$-differentiable and $h(x)=f(g(x))$. Then $h(x)$ is $\alpha$-differentiable, and for all $x$ with $x \neq 0$ and $g(x) \neq 0$, we have

$$
D_{x}^{\alpha} h(x)=D_{x}^{\alpha} f(g(x)) \cdot D_{x}^{\alpha} g(x) \cdot g(x)^{\alpha-1} .
$$

(vi) ( $\alpha$-integration by parts) Let $f, g:[a, b] \rightarrow \mathbb{R}$ be two functions such that $f g$ is differentiable. Then

$$
\int_{a}^{b} x^{\alpha-1} f(x) D_{x}^{\alpha} g(x) d x=\left.f(x) g(x)\right|_{a} ^{b}-\int_{a}^{b} x^{\alpha-1} g(x) D_{x}^{\alpha} f(x) d x
$$

Proof of Theorem 1.1 (i) For $\rho \equiv 1$, define

$$
y(x)=r(x) \sin (\sqrt{\lambda} \theta(x)) \quad \text { and } \quad D_{x}^{\alpha} y(x)=\sqrt{\lambda} r(x) \cos (\sqrt{\lambda} \theta(x)) .
$$

Taking

$$
D_{x}^{\alpha}\left(\frac{D_{x}^{\alpha} y(x)}{y(x)}\right)=D_{x}^{\alpha}\left(\frac{\sqrt{\lambda} \cos (\sqrt{\lambda} \theta(x))}{\sin (\sqrt{\lambda} \theta(x))}\right)
$$

and applying (1), one can obtain the phase equation

$$
\theta^{\prime}(x)=x^{\alpha-1}\left[1-\frac{q(x)}{\lambda} \sin ^{2}(\sqrt{\lambda} \theta(x))\right] .
$$

With each eigenvalue $\lambda_{n}$ of (1), one can associate the Prüfer angle $\theta_{n}(x) \equiv \theta\left(x ; \lambda_{n}\right)$ via (13) if one also specifies the initial condition

$$
\theta_{n}(0)=0, \quad n=1,2,3, \ldots
$$

In particular, $\theta_{n}(1)=n \pi$. Integrating (14) over $[0, x]$ with $\lambda=\lambda_{n}$, one can obtain

$$
\theta_{n}(x)=\frac{1}{\alpha} x^{\alpha}-\frac{1}{\lambda_{n}} \int_{0}^{x} t^{\alpha-1} q(t) \sin ^{2}\left(\sqrt{\lambda_{n}} \theta_{n}(t)\right) d t .
$$

Besides, integrating (14) over $[0,1]$ with $\lambda=\lambda_{n}$, one has

$$
\frac{n \pi}{\sqrt{\lambda_{n}}}=\frac{1}{\alpha}-\frac{1}{\lambda_{n}} \int_{0}^{1} x^{\alpha-1} q(x) \sin ^{2}\left(\sqrt{\lambda_{n}} \theta_{n}(x)\right) d x .
$$

That is,

$$
\sqrt{\lambda_{n}}=\alpha n \pi+\frac{\alpha}{\sqrt{\lambda_{n}}} \int_{0}^{1} x^{\alpha-1} q(x) \sin ^{2}\left(\sqrt{\lambda_{n}} \theta_{n}(x)\right) d x
$$




$$
\begin{aligned}
& =\alpha n \pi+\frac{\alpha}{2 \sqrt{\lambda_{n}}} \int_{0}^{1} x^{\alpha-1} q(x) d x-\frac{\alpha}{2 \sqrt{\lambda_{n}}} \int_{0}^{1} x^{\alpha-1} q(x) \cos \left(2 \sqrt{\lambda_{n}} \theta_{n}(x)\right) d x \\
& =\alpha n \pi+O\left(\frac{1}{n}\right)
\end{aligned}
$$

for sufficiently large $n$. Substituting (18) into (15), for sufficiently large $n$ one can obtain

$$
\sqrt{\lambda_{n}} \theta_{n}(x)=n \pi x^{\alpha}+O\left(\frac{1}{n}\right)
$$

Then the second integral term in (17) becomes

$$
\begin{aligned}
\int_{0}^{1} x^{\alpha-1} q(x) \cos \left(2 \sqrt{\lambda_{n}} \theta_{n}(x)\right) d x & =\int_{0}^{1} x^{\alpha-1} q(x) \cos \left(2 n \pi x^{\alpha}\right) d x+o(1) \\
& =\frac{1}{\alpha} \int_{0}^{1} q\left(s^{1 / \alpha}\right) \cos (2 n \pi s) d s+o(1) \\
& =o(1)
\end{aligned}
$$

for sufficiently large $n$ by the Riemann-Lebesgue lemma. Substituting (18) and (20) into (17), the Dirichlet eigenvalue asymptotic expansion (3) is valid.

(ii) For $q \equiv 0$, introduce another Prüfer-type substitution

$$
\lambda^{1 / 2} \rho^{1 / 2}(x) y(x)=R(x) \sin (\phi(x)) \quad \text { and } \quad D_{x}^{\alpha} y(x)=R(x) \cos (\phi(x)) .
$$

Taking

$$
D_{x}^{\alpha}\left(\frac{D_{x}^{\alpha} y(x)}{\lambda^{1 / 2} \rho^{1 / 2}(x) y(x)}\right)=D_{x}^{\alpha}\left(\frac{\cos (\phi(x))}{\sin (\phi(x))}\right)
$$

and applying (1), one can obtain

$$
\phi^{\prime}(x)=\lambda^{1 / 2} \rho^{1 / 2}(x) x^{\alpha-1}+\frac{1}{4}\left(\frac{\rho^{\prime}(x)}{\rho(x)}\right) \sin 2 \phi(x) .
$$

With each eigenvalue $\lambda_{n}$ of (1), one can associate the Prüfer angle $\phi_{n}(x) \equiv \phi\left(x ; \lambda_{n}\right)$ via (21) if one also specifies the initial condition

$$
\phi_{n}(0)=0, \quad n=1,2,3, \ldots
$$

In particular, $\phi_{n}(1)=n \pi$. Now integrating both sides of (22) with $\lambda=\lambda_{n}$, one can obtain

$$
n \pi=\lambda_{n}^{1 / 2} \int_{0}^{1} x^{\alpha-1} \rho^{1 / 2}(x) d x+\frac{1}{4} \int_{0}^{1}\left(\frac{\rho^{\prime}(x)}{\rho(x)}\right) \sin 2 \phi_{n}(x) d x .
$$

Note that if $\phi_{n}^{\prime}(x)=0$ is valid in some subinterval of $(0,1), \sin 2 \phi_{n}(x)$ will be constant in this subinterval. This implies that the function $\rho(x)$ depends on $\lambda_{n}$ in this subinterval from (22). This will contradict our original problem. Hence, the points satisfying $\phi_{n}^{\prime}(x)=0$ shall be 
isolated. Let $f(x)=\rho^{-1 / 2}(x)$, and then $-2 \rho^{1 / 2} f^{\prime}=\left(\frac{\rho^{\prime}}{\rho}\right)$. Note that, for sufficiently large $n$, by (22), one has

$$
\begin{aligned}
\frac{1}{4}\left(\frac{\rho^{\prime}}{\rho}\right) \sin 2 \phi_{n} & =\frac{1}{4}\left(-2 \rho^{1 / 2} f^{\prime}\right) \sin 2 \phi_{n} \frac{\phi_{n}^{\prime}}{\lambda_{n}^{1 / 2} \rho^{1 / 2} x^{\alpha-1}+\frac{1}{4}\left(-2 \rho^{1 / 2} f^{\prime}\right) \sin 2 \phi_{n}} \\
& =-\left(\frac{1}{2} \lambda_{n}^{-1 / 2} x^{1-\alpha} f^{\prime} \sin 2 \phi_{n}\right) \frac{\phi_{n}^{\prime}}{1-\frac{1}{2} \lambda_{n}^{-1 / 2} x^{1-\alpha} f^{\prime} \sin 2 \phi_{n}} \\
& =-\sum_{k=0}^{\infty}\left(\frac{1}{2} \lambda_{n}^{-1 / 2} x^{1-\alpha} f^{\prime} \sin 2 \phi_{n}\right)^{k+1} \phi_{n}^{\prime} \\
& =-\frac{\lambda_{n}^{-1 / 2}}{2} x^{1-\alpha} f^{\prime} \sin 2 \phi_{n} \cdot \phi_{n}^{\prime}+O\left(\frac{1}{\lambda_{n}^{1 / 2}}\right) .
\end{aligned}
$$

Then, by (23), for sufficiently large $n$, one gets

$$
\begin{aligned}
n \pi= & \lambda_{n}^{1 / 2} \int_{0}^{1} x^{\alpha-1} \rho^{1 / 2}(x) d x-\frac{\lambda_{n}^{-1 / 2}}{2} \int_{0}^{1} x^{1-\alpha} f^{\prime}(x) \sin 2 \phi_{n} \cdot \phi_{n}^{\prime} d x+O\left(\frac{1}{\lambda_{n}^{1 / 2}}\right) \\
= & \lambda_{n}^{1 / 2} \int_{0}^{1} x^{\alpha-1} \rho^{1 / 2}(x) d x-\frac{\lambda_{n}^{-1 / 2}}{2}\left(f^{\prime}(1)-\int_{0}^{1} \frac{d}{d x}\left[x^{1-\alpha} f^{\prime}(x)\right] \cos 2 \phi_{n} d x\right) \\
& +O\left(\frac{1}{\lambda_{n}^{1 / 2}}\right) \\
= & \lambda_{n}^{1 / 2} \int_{0}^{1} x^{\alpha-1} \rho^{1 / 2}(x) d x+O\left(\frac{1}{\lambda_{n}^{1 / 2}}\right) .
\end{aligned}
$$

Therefore, one can obtain the eigenvalue asymptotic

$$
\sqrt{\lambda_{n}} \int_{0}^{1} x^{\alpha-1} \rho^{1 / 2}(x) d x=n \pi+O\left(\frac{1}{n}\right)
$$

Remark 2.3 Assume that $\rho \equiv 1$. For the same issue for (1) coupling with the Neumann boundary conditions $D_{x}^{\alpha} y(0)=D_{x}^{\alpha} y(1)=0$, one can derive the similar expansion by the following arguments. From the phase equation (14), let $\lambda=\mu_{n}$ with $\theta_{n}(0)=\frac{\pi}{2}$ and $\theta_{n}(1)=$ $\left(n-\frac{1}{2}\right) \pi$. Applying the same calculation, one can obtain

$$
\sqrt{\mu_{n}}=\alpha(n-1) \pi+\frac{1}{2(n-1) \pi} \int_{0}^{1} x^{\alpha-1} q(x) d x+o\left(\frac{1}{n}\right)
$$

for sufficiently large $n$.

Before proving Theorem 1.2, we have to quote the fractional Rayleigh quotient result from [6].

Proposition 2.4 ([6, Lemma 12]) For the fractional eigenvalue problem (1) it holds that

$$
\lambda_{1}=\min _{y \neq 0} \frac{\int_{0}^{1} x^{\alpha-1}\left(D_{x}^{\alpha} y(x)\right)^{2} d x-\int_{0}^{1} x^{\alpha-1} q(x)(y(x))^{2} d x}{\int_{0}^{1} x^{\alpha-1}(y(x))^{2} d x} .
$$


Now it suffices to give the proof of Theorem 1.2.

Proof of Theorem 1.2 (i) By the eigenvalue estimates (3), one has

$$
\int_{0}^{1} x^{\alpha-1} q(x) d x=0
$$

Next, under the assumption, we will show that $\sin \left(\pi x^{\alpha}\right)$ is the first eigenfunction and then apply Proposition 2.4. Now $\sin \left(\pi x^{\alpha}\right)$ satisfies (2) and

$$
(\alpha \pi)^{2}=\lambda_{1} \leq \frac{\int_{0}^{1} x^{\alpha-1}\left(D_{x}^{\alpha} \sin \left(\pi x^{\alpha}\right)\right)^{2} d x-\int_{0}^{1} x^{\alpha-1} q(x) \sin ^{2}\left(\pi x^{\alpha}\right) d x}{\int_{0}^{1} x^{\alpha-1} \sin ^{2}\left(\pi x^{\alpha}\right) d x},
$$

where

$$
\begin{aligned}
& \int_{0}^{1} x^{\alpha-1}\left(D_{x}^{\alpha} \sin \left(\pi x^{\alpha}\right)\right)^{2} d x=\int_{0}^{1} x^{\alpha-1}(\alpha \pi)^{2} \cos ^{2}\left(\pi x^{\alpha}\right) d x=\frac{\alpha \pi^{2}}{2} \\
& \int_{0}^{1} x^{\alpha-1} q(x) \sin ^{2}\left(\pi x^{\alpha}\right) d x=\frac{1}{2}\left(\int_{0}^{1} x^{\alpha-1} q(x) d x-\int_{0}^{1} x^{\alpha-1} q(x) \cos \left(2 \pi x^{\alpha}\right) d x\right)=0
\end{aligned}
$$

and

$$
\int_{0}^{1} x^{\alpha-1} \sin ^{2}\left(\pi x^{\alpha}\right) d x=\frac{1}{2} \int_{0}^{1} x^{\alpha-1}\left(1-\cos \left(2 \pi x^{\alpha}\right)\right) d x=\frac{1}{2 \alpha}
$$

by (5) and (28). The above shows that $\sin \left(\pi x^{\alpha}\right)$ achieves the minimum value and is thus the first eigenfunction. Substituting this into (1), we obtain $q \equiv 0$ on $(0,1)$.

(ii) By (26),

$$
\int_{0}^{1} x^{\alpha-1} q(x) d x=0
$$

Let $y_{1}(x)$ be the first Neumann eigenfunction with $\mu_{1}=0$. One may assume, without loss of generality, that $y_{1}(x)>0$ on $[0,1]$. Taking into account the relation

$$
D_{x}^{\alpha}\left(\frac{D_{x}^{\alpha} y_{1}(x)}{y_{1}(x)}\right)=q(x)-\left(\frac{D_{x}^{\alpha} y_{1}(x)}{y_{1}(x)}\right)^{2}
$$

one can obtain

$$
\begin{aligned}
0 & =\int_{0}^{1} x^{\alpha-1} q(x) d x=\left[\frac{D_{x}^{\alpha} y_{1}(x)}{y_{1}(x)}\right]_{0}^{1}+\int_{0}^{1} x^{\alpha-1}\left(\frac{D_{x}^{\alpha} y_{1}(x)}{y_{1}(x)}\right)^{2} d x \\
& =\int_{0}^{1} x^{\alpha-1}\left(\frac{D_{x}^{\alpha} y_{1}(x)}{y_{1}(x)}\right)^{2} d x .
\end{aligned}
$$

Then $D_{x}^{\alpha} y_{1}(x)=0$ on $(0,1)$, and hence $y_{1}(x)$ is a constant on $(0,1)$. This implies $q \equiv 0$ on $(0,1)$. 
Funding

Not applicable.

\section{Abbreviations}

Not applicable

Availability of data and materials

Not applicable.

\section{Declarations}

\section{Ethics approval and consent to participate}

Not applicable.

\section{Consent for publication}

Not applicable.

\section{Competing interests}

The author declares that he has no competing interests.

\section{Authors' contributions}

The author wrote the draft and derived the theorem. He also read and approved the final manuscript.

\section{Publisher's Note}

Springer Nature remains neutral with regard to jurisdictional claims in published maps and institutional affiliations.

Received: 10 September 2021 Accepted: 5 December 2021 Published online: 17 December 2021

\section{References}

1. Abdelhakim, A.A., Machado, J.A.T.: A critical analysis of the conformable derivative. Nonlinear Dyn. 95, 3063-3073 (2019)

2. Abdeljawad, T.: On conformable fractional calculus. J. Comput. Appl. Math. 279, 57-66 (2015)

3. Adalar, I.: Ambarzumyan-type theorem for conformable Sturm-Liouville problem. In: 7th International Congress on Fundamental and Applied Sciences, Prishtina, Kosovo, 6 October (2020)

4. Adalar, I., Ozkan, A.S.: Inverse problems for a conformable fractional Sturm-Liouville operator. J. Inverse III-Posed Probl. 28(6), 775-782 (2020)

5. Adda, F.B., Cresson, J.: About non-differentiable functions. J. Math. Anal. Appl. 263(2), 721-737 (2001)

6. Al-Refai, M., Abdeljawad, T.: Fundamental results of conformable Sturm-Liouville eigenvalue problems. Complexity 2017, Article ID 3720471 (2017)

7. Anderson, D.R., Avery, R.I.: Fractional-order boundary value problem with Sturm-Liouville boundary conditions. Electron. J. Differ. Equ. 2015, 29 (2015)

8. Anderson, D.R., Camrud, E., Ulness, D.J.: On the nature of the conformable derivative and its applications to physics. J. Fract. Calc. Appl. 10(2), 92-135 (2019)

9. Babakhani, A., Daftardar-Gejji, V.: On calculus of local fractional derivatives. J. Math. Anal. Appl. 270(1), 66-79 (2002)

10. Batarfi, H., Losada, J., Nieto, J.J., Shammakh, W.: Three-point boundary value problems for conformable fractional differential equations. J. Funct. Spaces 2015, Article ID 706383 (2015)

11. Bayour, B., Torres, D.F.M.: Existence of solution to a local fractional nonlinear differential equation. J. Comput. Appl. Math. 321, 127-133 (2017)

12. Chen, Y., Yan, Y., Zhang, K.: On the local fractional derivative. J. Math. Anal. Appl. 362(1), 17-33 (2010)

13. Khalil, R., Al Horani, M., Yousef, A., Sababheh, M.: A new definition of fractional derivative. J. Comput. Appl. Math. 264, 65-70 (2014)

14. Kolwankar, K.M., Gangal, A.D.: Fractional differentiability of nowhere differentiable functions and dimensions, chaos: an interdisciplinary. J. Nonlinear Sci. 6(4), 505-513 (1996)

15. Mortazaasl, H., Akbarfam, A.J.: Trace formula and inverse nodal problem for a conformable fractional Sturm-Liouville problem. Inverse Probl. Sci. Eng. 28(4), 524-555 (2020)

16. Ortigueira, M.D., Martynyuk, V., Fedula, M., Machado, J.T.: The failure of certain fractional calculus operators in two physical models. Fract. Calc. Appl. Anal. 22(2), 255-270 (2019)

17. Ortigueira, M.D., Tenreiro Machado, J.A.: What is a fractional derivative? J. Comput. Phys. 293, 4-13 (2015)

18. Tarasov, V.E.: No violation of the Leibniz rule. No fractional derivative. Commun. Nonlinear Sci. Numer. Simul. 18(11), 2945-2948 (2013)

19. Tarasov, V.E.: No nonlocality. No fractional derivative. Commun. Nonlinear Sci. Numer. Simul. 62, 157-163 (2018)

20. Xiaojun, Y., Baleanu, D.: Fractal heat conduction problem solved by local fractional variation iteration method. Therm. Sci. 17(2), $625(2013)$

21. Zhao, D., Luo, M.: General conformable fractional derivative and its physical interpretation. Calcolo 54, $903-917$ (2017) 